\author{
Tesshu Otagiri $\cdot$ Kenji Sugai $\cdot$ Kazuki Kijima \\ Hiroko Arai · Yukio Sawaishi · Mitsuteru Shimohata \\ Kiyoshi Hayasaka
}

\title{
Periaxin mutation in Japanese patients with Charcot-Marie-Tooth disease
}

Received: 23 March 2006/ Accepted: 27 March 2006/Published online: 13 June 2006

(C) The Japan Society of Human Genetics and Springer-Verlag 2006

\begin{abstract}
Periaxin (PRX) plays an important role in the myelination of the peripheral nerve and consequently in the pathogenesis of Charcot-Marie-Tooth disease (CMT). To date, nine nonsense or frameshift $P R X$ mutations have been reported in eight families with CMT. The patients with $P R X$ mutations appeared to show characteristic clinical features with early onset but slow or no progression, a common result of mutations that lead to missing a C-terminal acidic domain. Here, we report a Japanese CMT patient with these characteristic clinical features, who was a compound heterozygote for $P R X$ R1070X and L132FsX153 mutations. We previously reported that three Japanese isolated families also had the homozygous R1070X mutation. To examine the potential founder effect of the R1070X mutation in the Japanese population, we performed haplotype analysis and found that each R1070X allele lay on a different haplotype background in these four families. Therefore, the high frequency of the R1070X mutation among the Japanese population is not likely the consequence of a founder effect, but probably a result of a mutation hot spot.
\end{abstract}

T. Otagiri $\cdot$ K. Kijima $\cdot$ H. Arai $\cdot$ K. Hayasaka $(\bowtie)$

Department of Pediatrics, Yamagata University

School of Medicine, 2-2-2 Iida-nishi, Yamagata

990-9585, Japan

E-mail: hayasaka@med.id.yamagata-u.ac.jp

Tel.: + 81-23-6285329

Fax: + 81-23-6285332

K. Sugai

Department of Child Neurology,

National Center Hospital for Mental, Nervous and Muscular

Disorders, National Center of Neurology and Psychiatry,

Tokyo, Japan

Y. Sawaishi

Department of Pediatrics,

Akita University School of Medicine, Akita, Japan

M. Shimohata

Department of Neurology, Brain Research Institute, Niigata University, Niigata, Japan
Keywords Periaxin $\cdot P R X \cdot$ Charcot-Marie-Tooth neuropathy $\cdot$ Peripheral nerve

\section{Introduction}

Charcot-Marie-Tooth disease (CMT) is characterized clinically by slowly progressive distal muscle atrophy and weakness, the absence of deep tendon reflexes, and pes cavus deformity of the foot. CMT has been classified into two types, the demyelinating form and the axonal form. At present, at least 11 different genes have been identified as causing demyelinating CMT, including autosomal dominant, autosomal recessive and X-linked types (Young and Suter 2003). Similar clinical manifestations are observed in patients carrying mutations in different genes, while variable presentations are present even in patients with an identical mutation in the same gene. Recently, there have been several reports that the periaxin (PRX) mutations were detected in the patients showing early onset of symptoms, but with slow or no progression (Boerkoel et al. 2001; Guilbot et al. 2001; Takashima et al. 2002; Kijima et al. 2004). We found heterozygous R1070X and L132fsX153 compound mutations of $P R X$ in another patient showing early onset but no progressive clinical symptoms. We further performed haplotype analysis at the $P R X$ locus in four Japanese families, three of which had been previously reported (Kijima et al. 2004).

\section{Materials and methods}

Patients

Case PRX-4

The patient was the second child of healthy, nonconsanguineous Japanese parents. She had a healthy elder brother and younger sister. The patient had shown gross motor delay since early infancy and walked without 
support at 5 years. A neurological examination at age 11 revealed mild distal muscle weakness and the absence of all deep tendon reflexes (Sawaishi et al. 1995). Touch and vibration senses were disturbed in a glove-stocking distribution. She walked and ran, stamping the floor. Cerebrospinal fluid protein was mildly elevated to $58 \mathrm{mg} / \mathrm{dl}$. The nerve conduction velocity of the right motor ulnar nerve was $2 \mathrm{~m} / \mathrm{s}$; no action potentials were evoked in other motor and sensory peripheral nerves. The biopsied sural nerve demonstrated markedly decreased numbers of myelinated fibers: 621 fibers $/ \mathrm{mm}^{2}$, none with a diameter greater than $5 \mu \mathrm{m}$. The electron micrographs showed numerous naked axons and thinly myelinated fibers, which were surrounded by atypical onion-bulbs consisting of double-layered empty basement membranes (Fig. 1a, b). At 26 years of age, she had no progressive worsening of disease and could walk and run, albeit unsteadily.
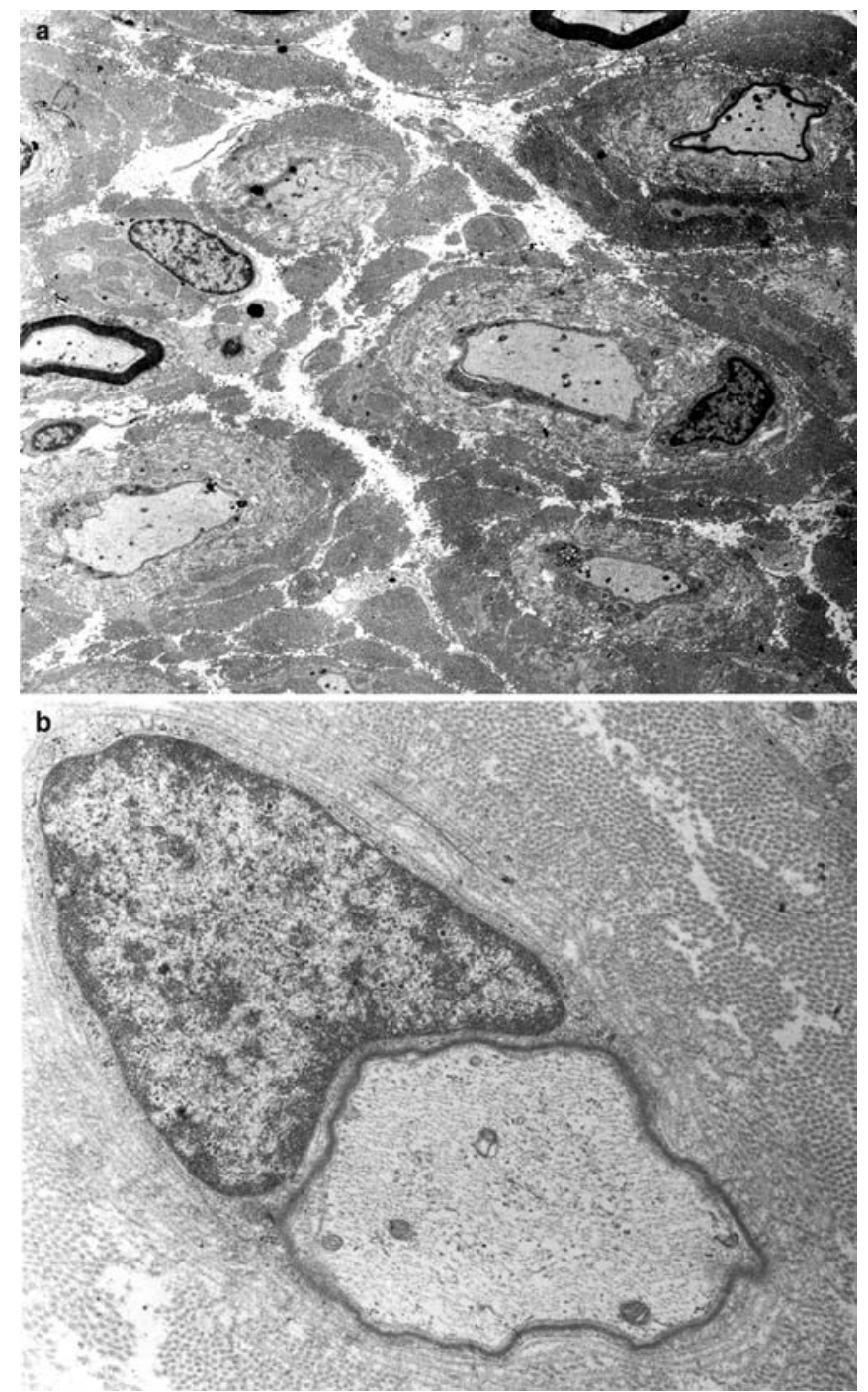

Fig. 1 a, b Electron microscopy. a Naked axons and thinly myelinated fibers are seen $(1,800 \times)$. b A thinly myelinated fiber is surrounded by atypical onion-bulbs consisting of double-layered empty basement membranes $(13,000 \times)$
Cases $P R X-1,2$ and 3

Cases PRX-1, 2 and 3 had early onset symptoms but slow or no progression, and were homozygous for the R1070X mutation, as described in our previous reports (Sawaishi et al. 1995; Kijima et al. 2004).

\section{Genetic analyses}

The Ethics Committee of the Yamagata University School of Medicine approved this study. With written informed consent from the patients and their families, peripheral blood specimens were used for genomic DNA extraction. All exons, including the exon-intron boundaries of $P R X$, were amplified using the polymerase chain reaction (PCR) with the primers designed based on the published sequences (Boerkoel et al. 2001). The mutations were screened by denaturing high-performance liquid chromatography analysis (DHPLC) (Transgenomic WAVE system). The fragments showing heteroduplex were sequenced using a BigDye Terminator Cycle Sequencing Reaction Kit (Applied Biosystems, Foster City, CA, USA) on an ABI PRISM Genetic Analyzer 310 (Applied Biosystems). The PCR fragments were also subcloned into a TA vector (Takara Bio, Tokyo, Japan) and sequenced for confirmation.

To determine the haplotype, the following microsatellite markers encompassing $P R X$ were analyzed: D19S217, D19S232, D19S421 and D19S881. CA repeat numbers were determined with an ABI PRISM Genetic Analyzer 310 using GeneScan Fragment Analysis (Applied Biosystem).

\section{Results}

DHLPC analysis revealed heteroduplexes in two PCR fragments that divided exon 7 into two. Sequencing of these fragments showed that the patient had the 3208C $>$ T (R1070X) and 394 395insATCCAGAGTC (L132FsX153) mutations (Fig. 2). Examination using parental DNA indicated that she inherited the R1070X mutation from her mother and the L132FsX153 mutation from her father. Neither of the mutations was detected in 100 healthy controls.

Haplotype analysis of $P R X$ showed that all $\mathrm{R} 1070 \mathrm{X}$ homozygous patients, except the sibling patients of family PRX-1, were also homozygous for markers. Each haplotype was different in the four families (Fig. 3). The patients of the family of PRX-1 were heterozygous for marker S881, suggesting that marker S881 is not within a haplotype block of $P R X$.

\section{Discussion}

We screened the $P R X$ mutation in a CMT patient with early onset symptoms but no progression and identified 
it as a compound heterozygote for $P R X$ R1070X and L132FsX153 mutations. Haplotype analysis in four Japanese families carrying the R1070X mutation revealed that each R1070X allele lay on a different haplotype.

The expression of L-periaxin in Schwann cells moves from the nucleus to the adaxonal or periaxonal cytoplasm, then to the abaxonal membrane of myelinating Schwann cells with the maturation of myelin (Scherer et al. 1995). L-periaxin on the abaxonal surface of myelinated Schwann cells forms a complex with dystrophin-related protein 2 linking the basal lamina outside the cell to the cytoskeleton within the cell (Scherer et al. 1995; Sherman and Brophy 2000; Sherman et al. 2001). This suggests that $P R X$ alters the role in the stage of myelination and plays significant roles in the formation and maintenance of myelin and in the signal transduction from the extracellular matrix to the cytoskeleton of myelinating Schwann cells. L-periaxin has four characteristic domains: PDZ, NLS, repeat and acidic domains (Gillespie et al. 1994; Sherman and Brophy 2000; Sherman et al. 2001). To date, nine pedigrees, carrying ten nonsense or frameshift mutations of $P R X$, have been reported (Boerkoel et al. 2001; Guilbot et al. 2001; Takashima et al. 2002; Kijima et al. 2004). It is interesting to note that all nine mutations are predicted to produce mutant proteins that lack an acidic domain. Acidic domains are known to mediate protein-protein interactions and the L-periaxin without an acidic domain may not be able to bind to the cytoskeleton of Schwann cells or to transmit the signals, leading to an impairment of stable myelin formation.
Early onset symptoms but with slow or no progression are remarkable clinical characteristics of patients with $P R X$ R1070X mutations. Several patients with $P R X$ mutations presented with severe symptoms and were diagnosed as having Dejerine-Sottas disease. Our four patients also showed prominent distal muscle weakness and atrophy in early infancy and were compatible with Dejerine-Sottas disease. However, their symptoms were slowly progressive or nonprogressive until four or five decades had passed. The R1070X mutation is the most downstream mutation registered so far and truncated proteins may contribute to the clinical course. In contrast, early onset and severely progressive phenotypes have been reported in patients carrying heterozygous MPZ (Hayasaka et al. 1993), PMP22 (Roa et al. 1993) or EGR2 (Timmerman et al. 1999) mutations. These mutant proteins exhibit dominant negative or gain-of-function behavior, whereby the patients display striking phenotypes. Meanwhile, $P R X$ mutations are expected to result in loss-of-function, which may explain the difference in the clinical phenotypes between $P R X$ mutations and $M P Z$, $P M P 22$ or $E G R 2$ mutations. Neurological dysfunction and clinical disability in demyelinating CMT are caused by loss or damage to large caliber axons. Damage to axons may progress slowly in patients with $P R X$ R1070X mutation.

We previously reported a homozygous R1070X mutation in three unrelated Japanese families (Kijima et al. 2004). The R1070X mutation might be one of the hotspots for single base-pair substitutions or may have been transmitted to Japanese patients due to founder effects. To investigate the potential founder
Fig. 2 Sequence determination revealed the $3208 \mathrm{C}>\mathrm{T}$

(R1070X) and

394 395insATCCAGAGTC

(L132FsX153) mutations in patient PRX-4. The sequence of the R1070X mutation was determined directly and that of the L132FsX153 mutation was determined after subcloning using TA vectors. The sites of mutations are underlined

$$
\text { control }
$$

394

A T C C A G A G T C T G T

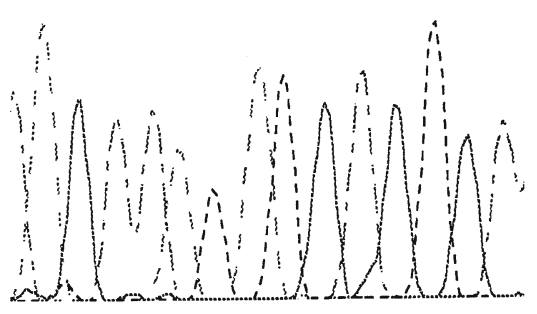

case PRX-4

394

A T C C A G A G T C A T C C A G A

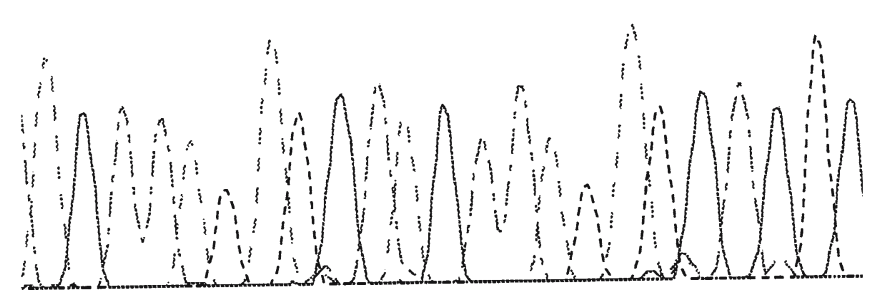

control

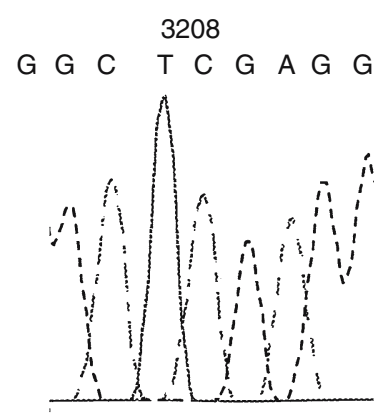

case PRX-4

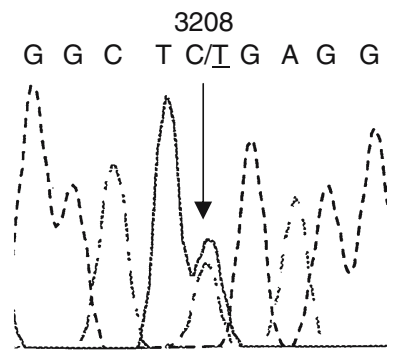




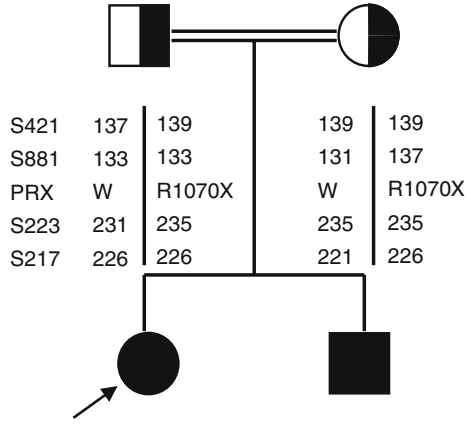

\begin{tabular}{ll|ll|l} 
S421 & 139 & 139 & 139 & 139 \\
S 881 & 133 & 137 & 133 & 137 \\
PRX & $R 1070 X$ & $R 1070 X$ & $R 1070 X$ & $R 1070 X$ \\
S223 & 235 & 235 & 235 & 235 \\
S217 & 226 & 226 & 226 & 226
\end{tabular}

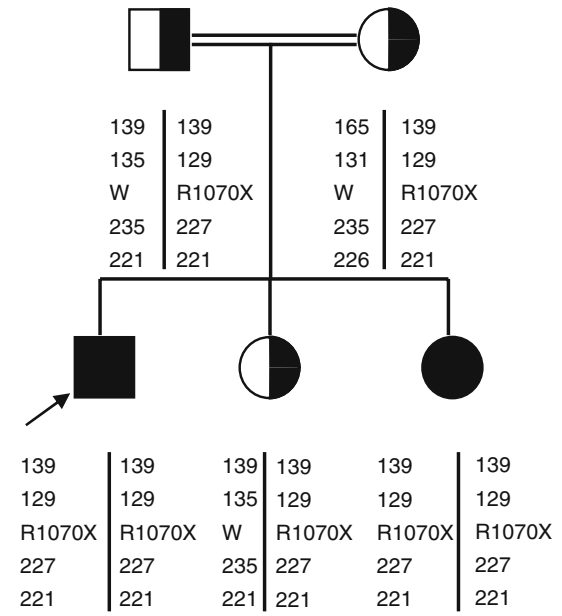

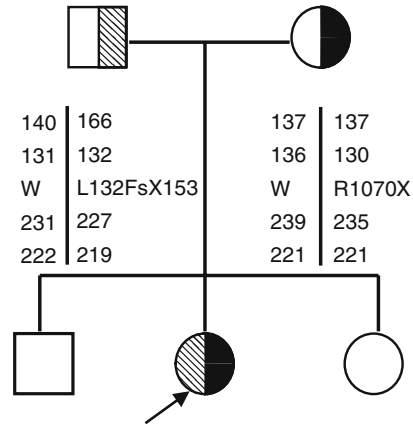

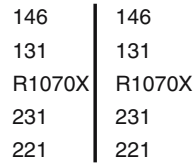

Fig. 3 Haplotype analysis was performed in four Japanese families with the PRX mutation. All families showed different haplotypes. Arrows indicate proposita and $w$ means wild type. In the family of

effect, we performed haplotype analysis in four families. As shown in Fig. 3, each R1070X allele lies on a different haplotype background in these four families. In addition, the R1070X mutation was also reported in a Turkish patient (Parman et al. 2004). The high frequency of the R1070X mutation within the Japanese population is not likely a consequence of a founder effect, but is probably a result of a mutation hot spot.

Information about the relationship between the genotype and phenotype, and about the genetic background of each race would contribute to medical genetics and molecular diagnosis.

Acknowledgements This work was supported by a grant from the Ministry of Education, Science, Culture and Sports of Japan, and a grant from the Ministry of Health, Labor and Welfare of Japan.

\section{References}

Boerkoel CF, Takashima H, Stankiewicz P, Garcia CA, Leber SM, Rhee-Morris L, Lupski JR (2001) Periaxin mutations cause recessive Dejerine-Sottas neuropathy. Am J Hum Genet 68:325-333

Gillespie CS, Sherman DL, Blair GE, Brophy PJ (1994) Periaxin, a novel protein of myelinating Schwann cells with a possible role in axonal ensheathment. Neuron 12:497-508

Guilbot A, Williams A, Ravise N, Verny C, Brice A, Sherman DL, Brophy PJ, LeGuern E, Delague V, Bareil C, Megarbane A, Claustres M (2001) A mutation in periaxin is responsible for CMT4F, an autosomal recessive form of Charcot-Marie-Tooth disease. Hum Mol Genet 10:415-421

Hayasaka K, Himoro M, Sawaishi Y, Nanao K, Takahashi T, Takada G, Nicholson GA, Ouvrier RA, Tachi N (1993) De novo mutation of the myelin P0 gene in Dejerine-Sottas disease (hereditary motor and sensory neuropathy type III). Nat Genet 5:266-268
PRX-4, the patient inherited the R1070X mutation from her mother and the L132FsX153 mutation from her father

Kijima K, Numakura C, Shirahata E, Sawaishi Y, Shimohata M, Igarashi S, Tanaka T, Hayasaka K (2004) Mutation causes early-onset but slow-progressive Charcot-Marie-Tooth disease. J Hum Genet 49:376-379

Parman Y, Battaloglu E, Baris I, Bilir B, Poyraz M, BissarTadmouri N, Williams A, Ammar N, Nelis E, Timmerman V, De Jonghe P, Necefov A, Deymeer F, Serdaroglu P, Brophy PJ, Said G (2004) Clinicopathological and genetic study of early-onset demyelinating neuropathy. Brain 127:2540-2550

Roa BB, Dyck PJ, Marks HG, Chance PF, Lupski JR (1993) Dejerine-Sottas syndrome associated with point mutation in the peripheral myelin protein 22 (PMP22) gene. Nat Genet 5:269273

Sawaishi Y, Hayasaka K, Goto A, Kawamura K, Ishiguro S, Sugai K, Nonaka I, Uyemura K, Takada G (1995) Congenital hypomyelination neuropathy: decreased expression of the $\mathrm{P} 2$ protein in peripheral nerve with normal DNA sequence of the coding region. J Neurol Sci 134:150-159

Scherer SS, Xu YT, Bannerman PG, Sherman DL, Brophy PJ (1995) Periaxin expression in myelinating Schwann cells: modulation by axon-glial interactions and polarized localization during development. Development 121:4265-4273

Sherman DL, Brophy PJ (2000) A tripartite nuclear localization signal in the PDZ-domain protein L-periaxin. J Biol Chem 275:4537-4540

Sherman DL, Fabrizi C, Gillespie CS, Brophy PJ (2001) Specific disruption of a Schwann cell dystrophin-related protein complex in a demyelinating neuropathy. Neuron 30:677-687

Takashima H, Boerkoel CF, De Jonghe P, Ceuterick C, Martin JJ, Voit T, Schroder JM, Williams A, Brophy PJ, Timmerman V, Lupski JR (2002) Periaxin mutations cause a broad spectrum of demyelinating neuropathies. Ann Neurol 51:709-715

Timmerman V, De Jonghe P, Ceuterick C, De Vriendt E, Lofgren A, Nelis E, Warner LE, Lupski JR, Martin JJ, Van Broeckhoven C (1999) Novel missense mutation in the early growth response 2 gene associated with Dejerine-Sottas syndrome phenotype. Neurology 52:1827-1832

Young P, Suter U (2003) The causes of Charcot-Marie-Tooth disease. Cell Mol Life Sci 60:2547-2560 